\title{
Selección dietaria de Tegula atra (Lesson, 1830) como una aproximación de preferencia sobre distintas especies de macroalgas en el sur de Chile
}

\author{
Dietary selection of Tegula atra (Lesson, 1830) as an approximation of \\ preference over different species of macroalgae from south of Chile
}

\author{
Ramona Pinochet ${ }^{1,3}$, Juan Carlos Soto ${ }^{2}$, Mauricio Palacios ${ }^{1,2,3}$ \& Sylvia Oyarzún ${ }^{2}$
}

\section{Resumen}

Entre las causas que determinan preferencia alimentaria se destacan las propiedades de las dietas del consumidor o de la interacción consumidor-presa. Debido al desafío que constituye incluir todos estos componentes para evaluar preferencia, es que se optó por una forma de medirla a través de la selección de un tipo de alimento en particular. Esta selección fue basada en la tasa de consumo del alimento, asumiendo que aquellos que satisfagan más rápido los requerimientos fisiológicos del organismo serán los que más se consuman. Si existe selección por un tipo de alimento en particular, la tasa de consumo sobre ese alimento será diferente si es presentado como mono dieta o dieta mixta. Con el fin de evaluar preferencia alimentaria en el caracol negro Tegula atra, se realizaron experimentos manipulativos de selectividad con individuos procedentes de dos localidades del sur del Chile: Bahía Metri (Puerto Montt) y Punta Santa Ana (Estrecho de Magallanes). Estos ejemplares fueron expuestos a mono dieta y dieta mixta de macroalgas. Los resultados de las tasas de consumo dan cuenta de la capacidad de este organismo de alimentarse de variadas especies de macroalgas, pero también de ciertos comportamientos selectivos, principalmente dado por el mayor consumo de la macroalga parda, Macrocystis pyrifera. Estos resultados concuerdan con la frecuencia en que se encuentran asociadas ambas especies en su ambiente natural.

\section{Palabras clave:}

herbivoria y relaciones tróficas, selección de dieta, tasas de consumo

\begin{abstract}
Among the causes that determine food preference are the properties of diets, consumer or consumerprey interactions. Due to the challenge of integrating all these causes to assess food preference, it was chosen a measuring the food selection the selection of a particular food type. The food selection was measured thoughout the consumption rate, assuming that those food that satisfy the body's physiological requirements more quickly, will be most consume. If there is selection for a particular type of food, consumption rate on that food will be different if it is presented as a mono- or mixeddiet. In order to evaluate dietary preference in the black snail Tegula atra, manipulative experiments of selectivity were carried out with individuals from two localities in Southern Chile: Bahía Metri (Puerto Montt) and Punta Santa Ana (strait of Magellan).
\end{abstract}

\footnotetext{
1 Programa de Doctorado en Biología Marina, Instituto de Ciencias Marinas y Limnológicas, Facultad de Ciencias, Universidad Austral de Chile. Edificio Pugin s/n Campus Isla Teja, Valdivia-Chile. $\measuredangle$ rjpinoch@gmail.com

2 Facultad de Ciencias, Universidad de Magallanes, Punta Arenas, Chile.

3 Centro Fondap de Investigación Dinámica de Ecosistemas Marinos de Altas Latitudes (IDEAL), Valdivia, Chile.
} 
The specimens were exposed to monospecific diet of macroalgae and macroalgae mixed-diet. The results of consumption rate showed the capacity of this organism to feed on varied species of macroalgae, but also certain selective behaviours, mainly due to the greater consumption of the macroalgae, Macrocystis pyrifera. These results coincide with the frequency of co-occurrence of both species on their natural environment.

\section{Key words:}

herbivory, trophic relationships, food selection, consumption rate

\section{INTRODUCCIÓN}

El patrón de alimentación de un organismo puede estar fuertemente influenciado por diversas causas, como las propiedades del alimento (disponibilidad y atracción), del consumidor (relaciones con otros organismos, hábitos alimentarios) o de la interacción consumidor-presa (Singer, 2000). Estas han sido categorizadas por Singer (2000) en tres componentes: aceptabilidad, como la probabilidad de adquirir la comida (relacionado a las características propias del alimento); selectividad, correspondiente a las proporciones de los diferentes alimentos en el medio ambiente (Ivlev, 1961) y la preferencia, como un componente que determina los patrones alimentarios de los organismos, y que está relacionada con la selección por parte de los consumidores. Estos tres componentes interactúan entre sí en el medio ambiente. Por lo tanto, no pueden ser analizados en forma independiente (Singer, 2000; Underwood et al. 2004). Una forma de medir la preferencia alimentaria es a través de la selección del tipo de alimento, en particular cuando este es presentado como única opción o junto a otras, y que se encentran disponibles en la misma abundancia (Underwood et al. op. cit.). Esta selección puede ser inferida a partir de la tasa de consumo del alimento, asumiendo: i) que aquellos alimentos que satisfagan más rápido los requerimientos fisiológicos del organismo serán los más consumidos (Peterson \& Bradley, 1978; Fairweather \& Underwood, 1983) y ii) que, si existe selección por un tipo de alimento en particular, este no será consumido en las mismas proporciones cuando se presenta sólo o junto a otras opciones (Underwood \& Clarke, 2005). Ciertas evaluaciones de preferencia alimentaria sobre un tipo de alimento han sido explicadas como consecuencia de asociaciones azarosas entre consumidor-presa, condicionadas principalmente por la disponibilidad del alimento en el ambiente natural. En macroinvertebrados marinos la disponibilidad de alimento en el ambiente tiene especial importancia, particularmente porque son organismos bentónicos sedentarios (Paine, 1969; Paine \& Vadas, 1969; Hoegh-Guldberg \& Pearse, 1995; Cheung \& Lam, 1999).

El caracol negro Tegula atra (Lesson 1830) es un gasterópodo de la familia Trochidae, que se distribuye por el Pacífico Sur oriental desde Pacasmayo, Perú ( $7^{\circ} 24^{\prime}$ S) hasta el estrecho de Magallanes, Chile (53 $28^{\prime}$ S) (Carcelles \& Williamson, 1951), mientras que por el lado Atlántico se han descrito poblaciones solo en las Islas Malvinas/ Falkland (Dall, 1910; Carcelles \& Williamson, 1951; Linse, 1999, 2002; Zagal, 2007). Forma extensas poblaciones en las playas rocosas del litoral, siendo un recurso potencialmente explotable por los bajos costos que implican su extracción (Coloma, 1974). A lo largo de su distribución, T. atra ha sido observada asociada a diferentes especies de macroalgas del orden Laminariales. En el norte de Chile, T. atra se encuentra en ambientes intermareales junto a Lessonia berteroana y en zonas submareales a Lessonia trabeculata (Veliz \& Vásquez, 2000; Oróstica et al. 2014). En otras áreas del sur de Chile, ha sido observada alimentándose principalmente de Macrocystis pyrifera (Vázquez \& Buschmann, 1997). Incluso existen registros de poblaciones de T. atra en el centro y sur de Chile asociadas a la macroalga roja, Mazzaella laminarioides (Buschmann et al. 1997). Los gasterópodos ramoneadores (Véliz \& Vásquez, 2000) presentan diferentes estrategias y preferencias en su alimentación, dependiendo de su morfología radular y la disponibilidad de alimento, entre otros factores (Reyes et al. 2001). En el caso de T. atra, su rádula ripidoglosa está principalmente adaptada para ramonear algas microscópicas y filamentosas (ver Reyes et al. op. cit.). Aun así, esta especie ha sido principalmente encontrada en la naturaleza asociada a macroalgas foliosas bentónicas, sugiriendo que la disponibilidad de alimento juega un rol clave en las preferencias de alimentación de esta especie. Sin 
embargo, se desconoce la existencia de otros factores externos que determinan su preferencia alimentaria, además de la disponibilidad de hábitat, de alimento y refugio contra depredadores (e.g. la calidad del alimento o selección por parte de consumidores). A pesar de su dieta restringida al nivel de individuo (Bolnick et al. 2002) y a su variada dieta a nivel de especie (Camus et al. 2013), hipotetizamos que T. atra posee una gran capacidad de extender sus hábitos de alimentación hacia el consumo de otras especies de macroalgas, y que no posee selección por una especie en particular. En este contexto el objetivo principal del estudio es evaluar mediante un experimento manipulativo la selección alimentaria (como una forma de evaluar la preferencia) de T. atra sobre distintas especies de macroalgas que presentan distintas abundancias en el litoral del sur de Chile.

\section{MATERIALES Y MÉTODOS}

\section{Sitios de colecta de organismos}

Se obtuvieron individuos de T. atra de dos localidades al sur de Chile: i) Bahía Metri $\left(45^{\circ} 35^{\prime}\right.$ 42,5" S; 72 42' 17,6" O), ubicada a $30 \mathrm{~km}$ del sureste de Puerto Montt $(n=20)$, ii) Sector de Punta Santa Ana (Estrecho de Magallanes, 53 37'17,6" S; $70^{\circ} 58^{\prime} 9,47^{\prime \prime}$ O) ubicada a $56 \mathrm{~km}$ del sur de Punta Arenas (Fig. 1) ( $n=20)$. La costa de Bahía Metri es un área protegida con amplitudes mareales de $7 \mathrm{~m}$ aproximadamente y con abundantes extensiones de sustrato arenoso, ideal para la proliferación de extensas poblaciones de macroalgas rojas como Gracilaria chilensis (Buschmann \& Goméz, 1993). Esta especie de macroalga constituye la principal fuente de alimento de T. atra, pero también se le ha observado alimentándose de otras macroalgas (e. g. Ulva sp. y M. pyrifera) En el sector de Punta Santa Ana las poblaciones de T. atra son submareales, habitando mayoritariamente sustrato de tipo macizo rocoso y asociado a frondosos bosques de Lessonia flavicans y $M$. pyrifera entre 1 a 10 metros de profundidad (Häussermann \& Försterra, 2010).

\section{Diseño experimental}

Se utilizaron tres especies de macroalgas como alimento, las cuales fueron extraídas desde Punta Santa Ana. Se seleccionaron Ulva sp.
(Chlorophyta), M. laminarioides es su estado vegetativo y reproductivo (Rhodophyta) y $M$. pyrifera (Ochrophyta, clase Phaeophyceae). Todas las muestras fueron trasladadas y aclimatadas en el Centro de Investigación para la Acuicultura y Recursos Marinos Subantárticos de la Facultad de Ciencias de la Universidad de Magallanes en Punta Arenas. La aclimatación y mantención de los individuos de T. atra y de las macroalgas, se realizó en cámaras de cultivo a una temperatura media de $10 \pm 1^{\circ} \mathrm{C}$, salinidad (34-35 psu) y un fotoperíodo $12: 12$, además de aireación constante. Con el objetivo de conferir la necesidad de apetencia al inicio del experimento, los individuos de $T$. atra fueron sometidos a un periodo de inanición, pues la condición directa desde el medio ambiente podría resultar en una interferencia en la respuesta normal a la preferencia del gasterópodo (Underwood et al. 2004).

El diseño experimental consistió en 5 acuarios de $30 \mathrm{~cm}$ de ancho x $40 \mathrm{~cm}$ de alto para cada grupo (bahía Metri y Punta Santa Ana). Cada acuario se subdividió en 4 unidades de cultivo de 15 $\mathrm{cm}$ de ancho $\mathrm{x} 20 \mathrm{~cm}$ de alto, con un volumen total de 4,5 litros de agua de mar filtrada a $1 \mu \mathrm{m}$ con aireación. En cada unidad se dispuso un ejemplar de T. atra que constituyó la unidad experimental. Para los ensayos se fijaron cinco tratamientos (4 tratamientos con monodieta con cada una de las especies de macroalgas y un tratamiento con dieta mixta incluyendo todas las especies de macroalgas seleccionadas). Cada unidad fue asignada a un tratamiento y cuatro réplicas por tratamiento. La importancia de incorporar tratamientos con monodieta es eliminar la posibilidad de selección y registrar la interacción que existe entre el depredador y la especie de macroalga en específico. Esta interacción está dada porque diferentes individuos de T. atra van a ser capaces de obtener y manejar algunas presas más rápidos que otras, y en consecuencia, probablemente se alimentarán más de una especie de macroalga que de la otra, incluso cuando las distintas ofertas de alimento sean elegidas al azar (Rapport \& Turner, 1970; Peterson \& Bradley, 1978; Fairweather \& Underwood, 1983; Liszka \& Underwood, 1990). Para alimentar a T. atra y homogenizar el tamaño de la macroalga suministrada, se utilizó un sacabocado con un diámetro interno fue de $16 \mathrm{~mm}$. 

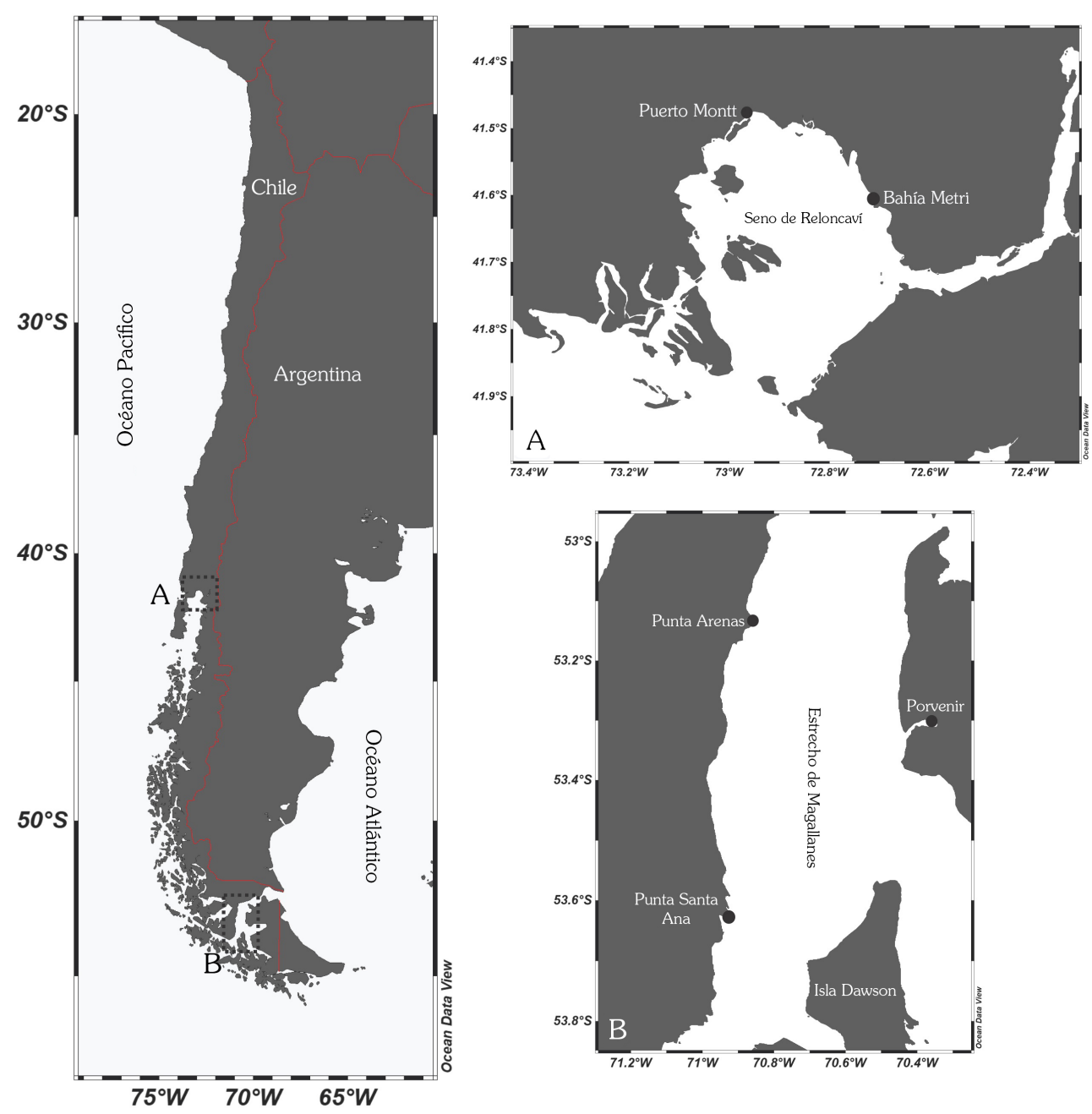

Fig. 1. Localización de las áreas de muestreo en el Sur de Chile. a) Ubicación de Bahía Metri, b) Ubicación de Punta Santa Ana.

Toma de datos y análisis estadísticos

La fase experimental del estudio tuvo una duración de 30 días, durante los cuales cada 2 días se removió la macroalga suministrada y se intercambió por un nuevo trozo. Posteriormente se registró la cantidad biomasa de macroalga consumida (g) por cada ejemplar de T. atra. El recambio del alimento apunta a minimizar los efectos de la descomposición (macroalgas) sobre los consumos finales (g). Los datos de consumo (g) del alimento se estandarizaron por peso (g) de Tegula (ya que existían individuos de tamaños distintos) y luego estos datos fueron transformados a tasas de consumo diaria (g/ $\left.\mathrm{dia}^{-1}\right)$. Se trabajó con los promedios de tasas de consumo obtenidos de las cuatro réplicas para cada tratamiento. Los datos fueron evaluados con una prueba de normalidad (Shapiro Wilks) y una prueba de homogeneidad de varianzas (Barlett), demostrando que los datos originales no seguían ninguno de estos dos supuestos. Para el caso de Punta Santa Ana, los datos de consumo fueron transformados a raíz cuadrada y en el caso de bahía Metri, transformados a raíz a la cuarta. Ambas transformaciones normalizaron y homogenizaron los datos, pudiendo realizar pruebas paramétricas para el análisis. Para las evaluaciones de selección dietaría, se realizaron pruebas $\mathrm{t}$ de Student independientes para cada especie de macroalga, con el fin de comparar los consumos en monodieta versus dieta mixta, además 
se realizaron pruebas t de Student independientes para todas las combinaciones pareadas de consumo sólo para dieta mixta.

\section{RESULTADOS}

De las ocho pruebas de t-Student realizados para las tasas de consumo diario (g/ $\mathrm{dia}^{-1}$ ) cuando las macroalgas eran ofrecidas en monodieta o dieta mixta, en dos casos las tasas de consumo ( $\mathrm{g} / \mathrm{dia}^{-1}$ ) durante monodieta y dieta mixta no fueron significativas en individuos de T. atra provenientes de bahía Metri para $M$. laminarioides en su estado reproductivo y Ulva ps. ( $p=0,14, p=0,76$, respectivamente) (Fig. 2 b y d). Las pruebas t-Student restantes para individuos de bahía Metri mostraron diferencias significativas en la tasa de consumo $\left(\mathrm{g} / \mathrm{dia}^{-1}\right)$ durante monodieta y dieta mixta para $M$. pyrifera y $M$. laminarioides vegetativa $(p=$ 0,0003, $p=0,002$, respectivamente) (Fig. 2 a y c). En el caso de los ejemplares de T. atra de Punta Santa Ana, las diferencias en las tasas de consumo diario cuando las macroalgas eran ofrecidas en monodieta o dieta mixta fueron significativas para todas las especies de alimento (M. laminarioides vegetativa, $p=0,02 ; M$. laminarioides reproductiva, $\mathrm{p}=0,005$; Ulva sp., $p=0,03 ;$ M. pyrifera, $p=0,002$, Fig. 3 a, $\mathrm{b}, \mathrm{c}, \mathrm{d})$. Para individuos de T. atra proveniente de ambas localidades, cuando se les ofreció la alternativa de dieta mixta, $M$. pyrifera fue la macroalga más consumida, seguida de $M$. laminarioides en su estado reproductivo y Ulva sp., las cuales fueron consumidas a la misma tasa (g/dia $\left.{ }^{-1}\right)$ (Fig. 4 a y b). Finalmente, la menos consumida fue $M$. laminarioides en estado vegetativa (Fig. 4 a y b). Más aún, es posible observar para ambas localidades que en general, independientemente si se le ofrecía a $T$. atra la misma cantidad de una especie en monodieta o dieta mixta, cuando era enfrentada a una dieta mixta, su tasa de consumo aumentó, excepto para el caso de $M$. laminarioides vegetativa, cuyo consumo disminuyó notablemente cuando era presentada en dieta mixta (Fig. 2c y 3c).
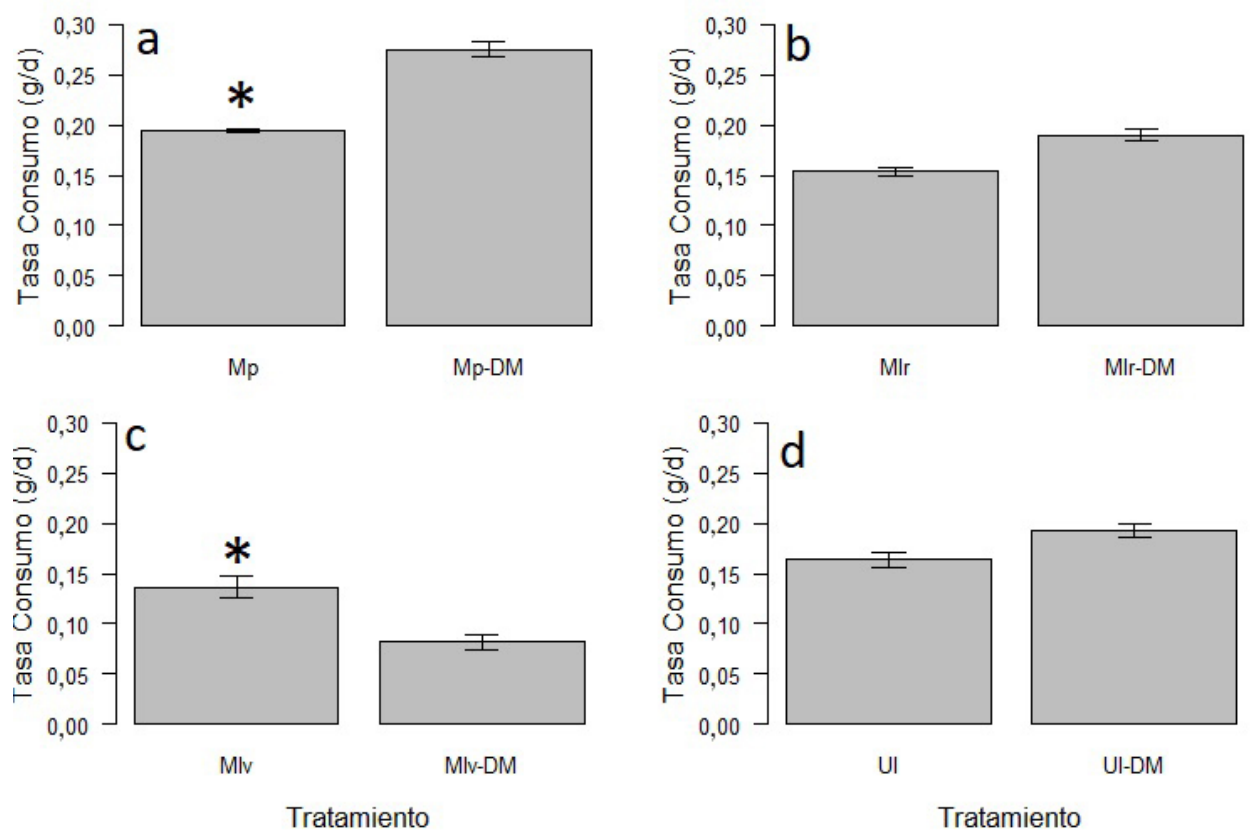

Fig. 2. Análisis independientes de Tasas de consumo $\left(\mathrm{g} / \mathrm{dia}^{-1}\right) \pm \mathrm{DE}$ de $T$. atra sobre los 4 tipos de dieta en mono dieta y dieta mixta para bahía Metri. Presencia de *, da cuenta de diferencias significativas. a) Mp, Mp-DM, M. pyrifera en mono y dieta mixta, respectivamente b) Mlr, Mlr-DM, M. laminarioides reproductiva en mono y dieta mixta, respectivamente c) Mlv, Mlv-DM, M. laminarioides vegetativa en mono y dieta mixta, respectivamente d) Ul, Ul-DM, Ulva sp. en mono y dieta mixta, respectivamente. 

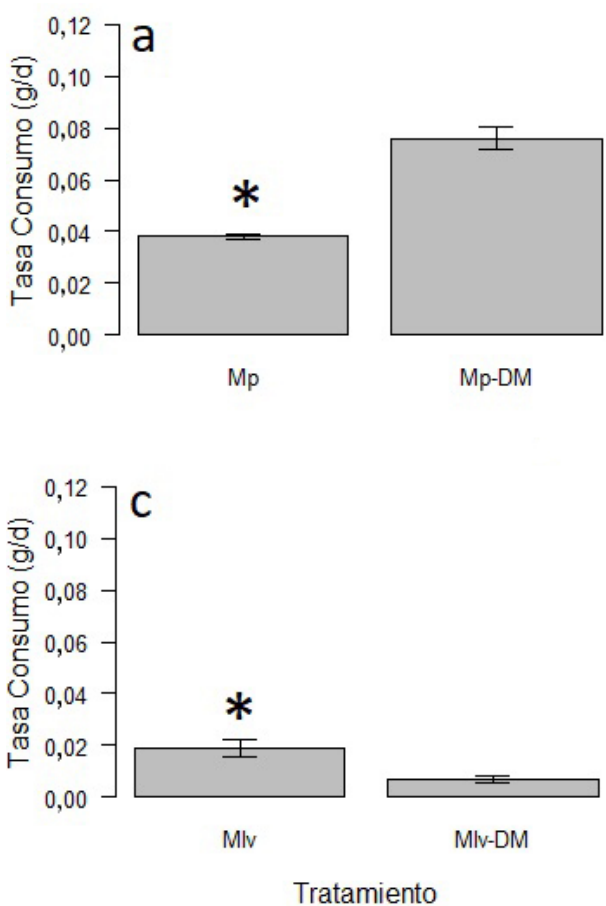
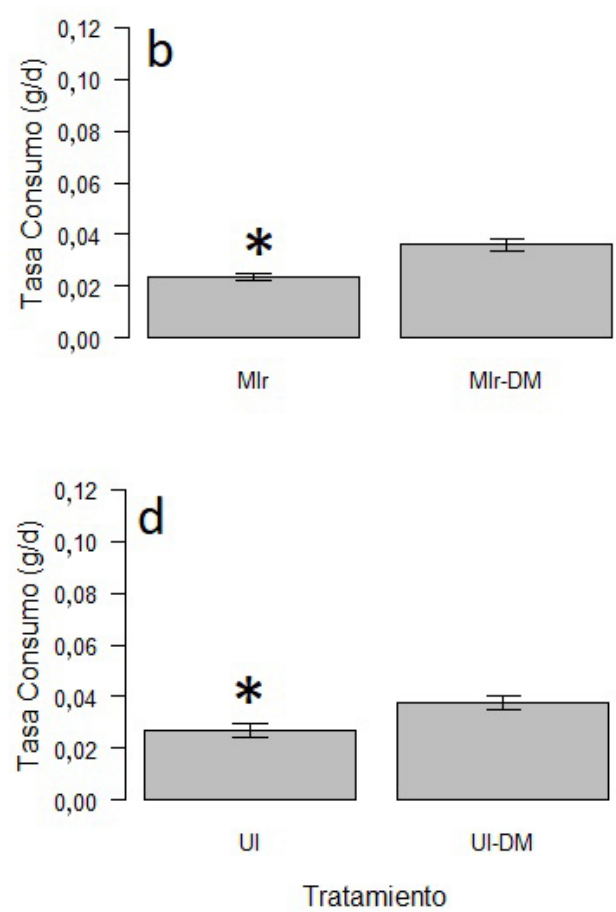

Fig. 3. Análisis independientes de Tasas de consumo $(\mathrm{g} / \mathrm{d}) \pm \mathrm{DE}$ de T. atra sobre los 4 tipos de dieta en mono dieta y dieta mixta para Punta Santa Ana. Presencia de *, da cuenta de diferencias significativas. a) Mp, MpDM, M. pyrifera en mono y dieta mixta, respectivamente b) Mlr, Mlr-DM, M. laminarioides reproductiva en mono y dieta mixta, respectivamente c) Mlv, Mlv-DM, M. laminarioides vegetativa en mono y dieta mixta, respectivamente d) Ul, Ul-DM, Ulva sp. en mono y dieta mixta, respectivamente.
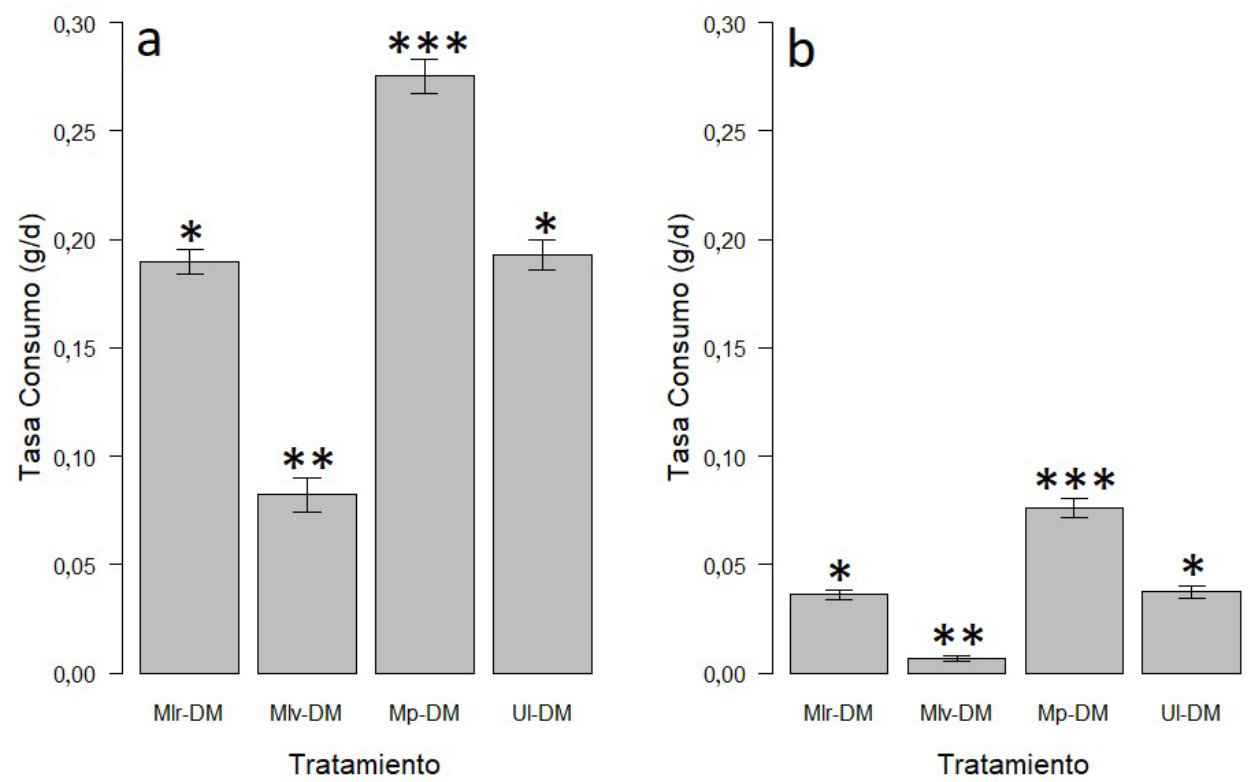

Fig. 4. Tasas de consumo (g/día $\left.{ }^{-1}\right) \pm \mathrm{DE}$ de $T$. atra sobre los 4 tipos de dieta en dieta mixta para a) Bahía Metri y b) Punta Santa Ana. Diferencias en *, representan diferencias significativas. 


\section{DISCUSIÓN}

Las diferencias encontradas entre las tasas de consumo en monodieta y dieta mixta dan cuenta de la selección que ejerce T. atra sobre distintos tipos de dieta, pudiendo ser negativa o positiva, entregando evidencia sobre el comportamiento alimentario de $T$. atra cuando es enfrentada a diferentes tipos de alimentos (macroalgas). Se sugiere en estos casos, que existe involucrada alguna decisión de pastoreo al momento de seleccionar que alimento consumir. En el caso de $M$. laminarioides en estado vegetativo, esta no fue seleccionada cuando se le presentaba junto a otras opciones de dieta. Esto podría estar explicado por la "hipótesis del balance entre crecimiento-diferenciación", que establece que tejidos reproductivos $y$ en crecimiento activo son defendidos, en menor medida, porque no poseen diferenciación celular en comparación al tejido vegetativo diferenciado (Herms \& Mattson, 1992). Aun así, esta hipótesis tiene dificultades para ser testeada y ha sido discutida en estudios posteriores (Cronin \& Hay, 1996). Otras causas que pudieran estar explicando el mayor consumo de tejido reproductivo tienen que ver con la mayor concentración de productos de la fotosíntesis como polisacáridos o como nitrógeno encontrados en tejidos reproductivos maduros (Gómez et al. 2007).

Para el resto de los tipos de dieta, en la mayoría de los casos se ejerció una selección positiva de T. atra sobre el alimento, pero aun así vemos que existen diferencias en la magnitud de selección. Las macroalgas difieren en sus contenidos nutricionales y calóricos. Por esta razón, diferencias en la calidad nutricional de distintas macroalgas presentes en el ambiente pueden ser también una causa influyente en la selectividad de un organismo sobre el consumo de una especie en particular. A partir de mediciones de componentes nutricionales se ha estimado que macroalgas rojas tienen mayor contenido de nitrógeno, contenido de carbohidratos solubles y contenido calórico disponible que macroalgas pardas y verdes; en cambio, macroalgas pardas poseen mayor contenido protéico (Granado \& Caballero, 2001). La producción de defensas químicas por algunas especies como consecuencia de herbívora también puede ser un factor importante en la selectividad del alimento ingerido (Toth \& Pavia, 2007). El mayor consumo de T. atra, en general, cuando era expuesta a dieta mixta puede estar relacionado también a señales químicas de las macroalgas. Este mecanismo originalmente es utilizado por las macroalgas para facilitar el consumo de los herbívoros por niveles tróficos más altos, pero también podría promover el movimiento de herbívoros en el ambiente y consecuentemente el consumo de alimento (Coleman et al. 1996; Coleman et al. 1997). En los ensayos con dieta mixta estas señales químicas se verían acentuadas por la mayor cantidad de alimento presentado y podrían estar influyendo en una mayor herbívora por parte de T. atra. Sin embargo, son necesarios estudios a futuro que involucren variables como contenido nutricional de los alimentos o señalización química entre presas para poder poner a prueba estas hipótesis.

Debido a que la composición macroalgal del hábitat de Punta Santa Ana está dominada por M. pyrifera, la asociación de T. atra a esta especie en particular no es considerada como preferencia alimentaria, sino es considerada como un factor azaroso debido a la disponibilidad de alimento y hábitat. Esta es una razón importante para decidir realizar experimentos manipulativos en laboratorio, ya que al proporcionar las mismas condiciones a todos los individuos estamos evitando estas desviaciones que nos podrían llevar a mal interpretar hábitos de preferencia alimentaria. Debido al mayor consumo de $M$. pyrifera por los individuos de T. atra de ambas localidades durante los ensayos experimentales, se sugiere que, si bien no es la única especie seleccionada, existe una alta presión selectiva sobre esta la que estaría explicada por los hábitos alimentarios de T. atra en el ambiente, o por los aportes nutricionales que esta le proporciona.

\section{CONCLUSIÓN}

En el caso de este estudio, luego de observaciones previas en terreno y de los experimentos manipulativos, se sugiere que la dieta de T. atra se encuentra compuesta de varias especies de macroalgas. Sin embargo, como consecuencia de diversas causas, existe 
una tendencia a alimentarse mayormente de M. pyrifera. A pesar de que la evaluación de selección en este caso nos permitió hacernos una idea de los patrones alimentarios de T. atra en relación con sus preferencias, existen algunos detalles que podrían mejorarse o perfeccionarse para la obtención de resultados más certeros. Dentro de estas modificaciones están el aumento del número muestreal, perfeccionamiento del diseño experimental para lograr, por ejemplo, obtener mayor poder estadístico de los datos o la inclusión de otras variables medidas como sexo o edad de los individuos de T. atra, composición química de las dietas y controles para evaluar crecimiento ontogénico de las macroalgas, lo que podría influir en el peso del alimento y llevar a subestimación del alimento ingerido. En cuanto a la importancia o aplicabilidad de resultados como este, se encuentran la evaluación de plasticidad del comportamiento alimentario hacia hábitos más generalistas, asociados a una mayor resiliencia de las poblaciones ante perturbaciones (Briscoe \& Sebens, 1988) o la generación de información especie específica útil para ser usada en actividades de cultivo o establecimiento de normas regulación, asociado a un manejo sustentable del recurso.

\section{LITERATURA CITADA}

Aguilera-Muñoz, F., Lafarga-Cruz, F., \& GallardoEscárate, C. (2009). Molecular analysis in Chilean commercial gastropods based on $16 \mathrm{~S}$ rRNA, COI and ITS1-5.8 S rDNAITS2 sequences. Gayana, 73(1), 17-27.

Bolnick, D. I., Yang, L. H., Fordyce, J. A., Davis, J. M., \& Svanbäck, R. (2002). Measuring individual-level resource specialization. Ecology, 83(10), 2936-2941.

Briscoe, C. S., \& Sebens, K. P. (1988). Omnivory in Strongylocentrotus droebachiensis (Miiller) (Echinodermata: Echinoidea): predation on subtidal mussels. Journal of Experimental Marine Biology and Ecology, 115,1-24.

Buschmann, A. H., \& Gómez, P. (1993). Interaction mechanisms between Gracilaria chilensis (Rhodophyta) and epiphytes. Hydrobiologia, 260(1), 345-
351.

Buschmann, A. H., Correa, J. A., Beltrán, J., \& Retamales, C. A. (1997). Determinants of disease expression and survival of infected individual fronds in wild populations of Mazzaella laminarioides (Rhodophyta) in central and southern Chile. Marine Ecology Progress Series, 154, 269-280.

Carcelles, A., \& Williamson, S. (1951). Catálogo de los moluscos marinos de la provincia Magallánica. Revista del Instituto Nacional de Investigación y Ciencias Naturales, 2, 225-383.

Camus, P. A., Arancibia, P. A., \& Ávila-Thieme, I. (2013). Una caracterización trófica de los consumidores intermareales en costas rocosas de Chile. Revista de Biología Marina y Oceanografía, 48(3), 431-450.

Cheung, S. G., \& Lam, S. (1999). Effect of food availability on egg production and packaging in the intertidal scavenging gastropod Nassarius festivus. Marine Biology, 135(2), 281-287.

Coleman, R. A., Barker, A. M., \& Fenner, M. (1996). Cabbage (Brassica oleracea var. Capitata) fails to show wound-induced defence against a specialist and a generalist herbivore?. Oecologia, 108(1), 105-112.

Coleman, R. A., Barker, A. M., Fenner, M., \& King, F. C. (1997). Relative effect of different host feeding site on long-range host location and electroantennogram response in the parasitoid Cotesia glomerata (Hym., Braconidae). Journal of Applied Entomology, 121(1-5), 487494.

Coloma, L. (1974). Estudio histológico de la gónada de Tegula (Chlorostoma) atra (Lesson, 1830), (Mollusca, Gastrópoda, Trochidae). Boletín de la Sociedad Biológica de Concepción, 48, 359-363.

Cronin, G., \& Hay, M. E. (1996). Within-plant variation in seaweed palatability and chemical defenses: optimal defense theory versus the growth-differentiation balance hypothesis. Oecologia, 105(3), 361-368.

Dall, W. (1910). Reporto an Collection of shells from Perú, with a summary of the Peruvian zoological. Proceedings of the United 
States National Museum, 37(176), 239.

Fairweather, P. G., \& Underwood, A. J. (1983).

The apparent diet of predators and biases due to different handling times of their prey. Oecologia, 56(2-3), 169-179.

Gómez, I., Orostegui, M., \& Huovinen, P. (2007). Morpho-functional patterns of photosynthesis in the south pacific kelp Lessonia nigrescens: effects of UV radiation on $14 \mathrm{c}$ fixation and primary photochemical reactions. Journal of Phycology, 43(1), 55-64.

Granado, I., \& Caballero, P. (2001). Feeding rates of Littorina striata and Osilinus atratus in relation to nutritional quality and chemical defenses of seaweeds. Marine Biology, 138(6), 1213-1224.

Häussermann, V., \& Försterra, G. (2010). Fauna Marina Bentónica de la Patagonia Chilena Guía de identificación ilustrada. Santiago de Chile: Nature in Focus.

Herms, D. A., \& Mattson, W. J. (1992). The dilemma of plants: to grow or defend. Quarterly review of biology, 283-335.

Hoegh-Guldberg, O. V. E., \& Pearse, J. S. (1995). Temperature, food availability, and the development of marine invertebrate larvae. American Zoologist, 35(4), 415425.

Ivlev, V. S. (1961). Experimental Ecology of the Feeding of Fish. New York: Yale University Press.

Linse, K. (1999). Mollusca of the Magellan region. A checklist of the species and their distribution. In: Magellan-Antarctic: Ecosystems that drifted apart. W. E. Arntz \& C. Ríos (Eds.). Scientia Marina, 63(Supl.1), 399-407.

Linse, K. (2002). The shelled Magellanic Mollusca: with special reference to biogeographic relations in the Southern Ocean. Theses Zoologicae, 34. A.R.G. Gantner: Ruggell

Liszka, D., \& Underwood, A. J. (1990). An experimental design to determine preferences for gastropod shells by a hermit-crab. Journal of Experimental Marine Biology and Ecology, 137(1), 47-62.
Oróstica, M., Aguilera, M. A., Donoso, G., Vásquez, J., \& Broitman, B. R. (2014). Effect of grazing on distribution and recovery of harvested stands of Lessonia berteroana kelp in northern Chile. Marine Ecology Progress Series, 511, 71-82.

Paine, R. T. (1969). The Pisaster-Tegula Interaction: Prey Patches, Predator Food Preference, and Intertidal Community Structure. Ecology, 50(6), 950-961.

Paine, R. T., \& Vadas, R. L. (1969). The effects of grazing by sea urchins, Strongylocentrotus spp., on benthic algal populations. Limnology and Oceanography, 14, 710- 719.

Peterson, C. H., \& Bradley, B. P. (1978). Estimating the diet of a sluggish predator from field observations. Journal of the Fisheries Board of Canada, 35(1), 136141.

Rapport, D. J., \& Turner, J. E. (1970). Determination of predator food preferences. Journal of Theoretical Biology, 26(3), 365-372.

Reyes, Y., Córdova, C., Romero, L., \& Paredes, C. (2001). Marcas radulares producidas por gasterópodos pastoreadores del intermareal rocoso. Revista Peruana de Biología, 8(1), 38-44.

Singer, M. C. (2000). Reducing ambiguity in describing plant-insect interactions:" preference", "acceptability" and" electivity". Ecology Letters, 3(3), 159162.

Toth, G. B., \& Pavia, H. (2007). Induced herbivore resistance in seaweeds: a metaanalysis. Journal of Ecology, 95(3), 425434.

Underwood, A. J., \& Clarke, K. R. (2005). Solving some statistical problems in analyses of experiments on choices of food and on associations with habitat. Journal of Experimental Marine Biology and Ecology, 318(2), 227-237.

Underwood, A. J., Chapman, M. G., \& Crowe, T. P. (2004). Identifying and understanding ecological preferences for habitat or prey. Journal of Experimental Marine Biology and Ecology, 300(1), 161-187. 
Vásquez, J. A., \& Buschmann, A. H. (1997). Herbivore-kelp interactions in Chilean subtidal communities: a review. Revista Chilena de Historia Natural, 70, 41-52. Veliz, D., \& Vásquez, J. A. (2000). La familia Trochidae (Mollusca: Gastropoda) en el norte de Chile: consideraciones ecológicas y taxonómicas. Revista Chilena de Historia Natural, 73(4), 757-769.

Zagal, C. \& Hermosilla, C. (2007). Guía de invertebrados Marinos. Segunda Edición. Punta Arenas: Editorial Fantástico Sur. 